Trauma Berufskrankh 2008 10 [Suppl 2]:263-266

DOI 10.1007/s10039-007-1337-x

Online publiziert: 20. April 2008

(c) Springer Medizin Verlag 2008

\author{
H. Nolting \\ Landesverband Nordostdeutschland der gewerblichen Berufsgenossenschaften, Berlin
}

\title{
BG-Aktuell
}

Die gesetzliche Unfallversicherung verändert sich im Eiltempo - zumindest gemessen an ihrer traditionsreichen Geschichte. In diesen Monaten wurden und werden voraussichtlich Entscheidungen getroffen, die unsere Organisation und auch das Leistungsrecht grundlegend verändern. Der vorliegende Beitrag soll chronologisch an die aktuelle Situation heranführen:

Noch unter Federführung der alten Bundesregierung wurde 2005 eine BundLänder-Arbeitsgruppe zur Reform der gesetzlichen Unfallversicherung eingesetzt bezeichnenderweise ohne diese selbst $\mathrm{zu}$ beteiligen. Ergebnis war ein Mitte 2006 von der Arbeitsgruppe verabschiedetes Eckpunktepapier, das folgende Aussagen traf:

- Die Zahl der Berufsgenossenschaften soll durch so genannte intelligente Fusionen auf 6 reduziert werden. Es soll grundsätzlich nur noch eine Unfallkasse pro Bundesland geben.

- Es soll eine einheitliche Spitzenkörperschaft für Unfallkassen und Berufsgenossenschaften mit staatlicher Aufsicht gebildet werden; diese soll umfassende Weisungs- und Eingriffsrechte gegenüber den Berufsgenossenschaften erhalten.

- Weil diese Maßnahmen angeblich Verwaltungskosten einsparen, soll der Gesetzgeber den Berufsgenossenschaften hierzu eine Vorgabe machen: $20 \%$ der Kosten sind innerhalb von 5 Jahren einzusparen.

- Im Leistungsrecht soll die Zielgenauigkeit der Rentenzahlungen erhöht werden (zur Erläuterung s. unten) Rente soll grundsätzlich nur noch bis zur Altersgrenze gezahlt werden. Wegeunfälle sollen weiter entschädigt werden.
Während in der Folgezeit die Politiker diskutierten und im Ministerium an einem Gesetzentwurf gearbeitet wurde, haben die Berufsgenossenschaften gehandelt. Die Mitgliederversammlung des Hauptverbands der gewerblichen Berufsgenossenschaften traf im Dezember 2006 aus dem Ehrenamt heraus und somit aus eigener Kraft wegweisende Beschlüsse.

\section{Organisation und Finanzierung}

\section{Fusionen}

Die Berufsgenossenschaften fusionieren und bilden nach einer angemessenen Übergangsfrist 9 neue Berufsgenossenschaften: Wir sind durchaus auch der Auffassung, dass es zu viele Unfallversicherungsträger gibt. Bei der notwendigen Konzentration muss aber immer noch das Branchenprinzip erkennbar bleiben. Wir verdanken dieser Differenzierung unsere Erfolge in der Prävention und die Akzeptanz in der Wirtschaft.

Auf dem Weg zu diesem neuen Modell haben bereits verschiedene Berufsgenossenschaften ihre Fusion vollzogen, beschlossen oder stehen unmittelbar davor. Der Gesetzgeber hat diese Selbstverpflichtung der Berufsgenossenschaften akzeptiert. Er hat in den aktuellen Entwurf eines Unfallversicherungsreformgesetzes die Zahl 9 übernommen. Hierzu gibt es also mittlerweile Konsens.

Um die verbliebenen Berufsgenossenschaften besser voneinander abgrenzen zu können, haben die Berufsgenossenschaften darüber hinaus verschiedene Vorschläge an den Gesetzgeber beschlossen. Eine Schlichtungsstelle wird künftig verhindern, dass Berufsgenossenschaften in Zuständigkeitsfragen gegeneinander klagen.

\section{Spitzenkörperschaft}

Auch die politische Forderung nach Bildung eines gemeinsamen Dachverbands wurde von den Unfallversicherungsträgern aufgegriffen und kurzfristig umgesetzt. Der HVBG und der Bundesverband der Unfallkassen fusionierten zum Verband der „Deutschen gesetzlichen Unfallversicherung“, kurz DGUV. Eingriffe in die Aufbau- und Ablauforganisation der Berufsgenossenschaften sind dem neu gebildeten Verband nach seiner Satzung allerdings nicht möglich. Auch handelt es sich bei ihm um einen eingetragenen Verein, wie das schon bisher bei beiden Spitzenverbänden der Fall war.

Es ist noch nicht klar, ob die Politik an ihrer Forderung der Verstaatlichung des Spitzenverbands festhält. Im bereits erwähnten Arbeitsentwurf des BMAS ist die Verkörperschaftung unverändert enthalten. Arbeitgeber- und Arbeitnehmervertreter haben jedoch deutlich zum Ausdruck gebracht, dass sie in der Lage und willens sind, ihre Angelegenheiten selbst zu regeln und staatlichen Dirigismus und Einfluss hier ablehnen. Es muss der Grundsatz „Vorfahrt der Selbstverwaltung" gelten.

\section{Finanzierung}

Die solidarische Umlagefinanzierung der Berufsgenossenschaften hat sich grundsätzlich bewährt. Problematisch wurde es in der Vergangenheit jedoch immer, wenn infolge eines Strukturwandels eine abnehmende Zahl von Unternehmen die Belastung für Renten aus wirtschaftlich besseren Tagen tragen musste. Es waren diese Verwerfungen, die letztlich zur Fusion der Bau-Berufsgenossenschaften führten. Die Veränderungen in der Struktur der Unternehmerschaft hat auch der 
Lastenausgleich nicht auffangen können. Die Solidargemeinschaften wurden einfach zu klein. Diese Frage aber grundsätzlich, so wie es das Eckpunktepapier vorsieht, ausschließlich über so genannte „intelligente Fusionen“ von Berufsgenossenschaften angehen zu wollen, ist weder besonders zukunftsweisend noch effizient. Daher haben die Berufsgenossenschaften beschlossen, diesem Problem grundsätzlicher zu begegnen und dem Gesetzgeber eine Änderung der Finanzierung vorzuschlagen:

Jede Berufsgenossenschaft trägt $\mathrm{zu}$ nächst nur Rentenlasten in einer Höhe, die der Zahl der Arbeitsunfälle und Berufskrankheiten in ihrer Branche entspricht. Was über diese Belastung hinausgeht, die so genannte Überaltlast, tragen alle Berufsgenossenschaften gemeinsam. Dies würde dazu führen, dass zukünftig etwa 30\% aller Rentenlasten als Überaltlast umverteilt werden würden. Belastet wären die boomenden Dienstleistungsbranchen, also z. B. die Verwaltungs-BG und die BGW, entlastet werden würden das produzierende Gewerbe. Der Gesetzgeber hat zugesagt, diese Lösung aufzugreifen und die Finanzierungsregelungen entsprechend zu ändern. Sie sind Bestandteil des aktuellen Gesetzesentwurfs.

\section{Resümee}

Für Organisation und Finanzierung würde es ausreichen, wenn der Gesetzgeber die Vorschläge der Unfallversicherungsträger aufgreifen und umsetzen würde.

\section{Leistungsrecht}

Dieses im sozialen Konsens zu reformieren, erscheint deutlich schwieriger.

Eine wesentliche Zielrichtung der Reform ist es, wie vorgetragen, die Zielgenauigkeit der Rentenzahlungen zu verbessern. Man hält es für reformbedürftig, dass die Minderung der Erwerbsfähigkeit abstrakt berechnet wird, also ohne Rücksichtnahme auf eine tatsächliche Verdienstminderung im Einzelfall. Und in der Tat ist es richtig, dass sich die Arbeitswelt und die medizinische und berufliche Rehabilitation weiterentwickelt haben. In vielen Fällen ist eine Weiterbeschäftigung bei gleichem Einkommen möglich. Um- gekehrt ist derjenige $u$. U. individuell benachteiligt, der eine spürbare Einkommensminderung zu verzeichnen hat oder vielleicht sogar arbeitslos wird.

\section{Konkrete Planungen des Arbeits- und Sozialministeriums}

\section{Aufspaltung der Rente}

Vorgesehen ist, die Rente in einen materiellen und einen immateriellen Teil aufzuspalten.

Materieller Teil der Rente. Der konkrete Erwerbsschaden wird durch eine so genannte Erwerbsminderungsrente aufgefangen. Zur Berechnung wird das Einkommen vor dem Unfall mit dem erzielbaren Einkommen nach dem Unfall in Relation gesetzt. Daraus ergibt sich die Höhe der Erwerbsminderung in \%.

$\mathrm{Ab}$ 10\% Erwerbsminderung soll es künftig einen Rentenanspruch geben. Wie bisher würde dieser Prozentsatz mit dem ermittelten Jahresarbeitsverdienst multipliziert werden. 60\% hiervon wären zukünftig die Vollrente (bisher sind dies 2/3). Vorgesehen ist, bei Arbeitslosigkeit höhere Renten zu zahlen, was zum Ruhen des Anspruchs auf Arbeitslosengeld führen würde. Hier sollen zukünftig Lasten von der Bundesagentur für Arbeit auf die Unfallversicherung verlagert werden.

Grundsätzlich soll der Anspruch auf Erwerbsminderungsrente nach dem Arbeitsentwurf mit dem Erreichen der Regelaltersgrenze enden. Zum Ausgleich muss der Unfallversicherungsträger aus der Erwerbsminderungsrente entsprechende Beiträge an die Deutsche Rentenversicherung zahlen.

Immaterieller Teil der Rente. Über die Erwerbsminderungsrente hinaus ist vorgesehen, den immateriellen Schaden durch eine neue Leistung, den so genannten Gesundheitsschadensausgleich, zu ersetzen. Insbesondere Schwerverletzte erhielten hierdurch höhere Leistungen. Die Bemessung soll in Anlehnung an das Versorgungsrecht nach dem „Grad der Schädigungsfolgen“ (GdS) erfolgen. Hierzu werden, abgestuft nach einem 10\%-Schema, monatliche Entschädigungsbeträge festgelegt. Die Untergrenze für Entschädigungen soll bei einem Grad der Schä- digungsfolgen von 30\% liegen. Im Gegensatz zur Erwerbsminderungsrente soll der Gesundheitsschadensausgleich so lange gezahlt werden, wie die Schädigung vorhanden ist, also u. U. ein Leben lang.

\section{Rehabilitation}

Als weiteren wesentlichen Punkt nannte der Gesetzentwurf den Ausbau der beruflichen Rehabilitation einschließlich der Arbeitsvermittlung. Er geht davon aus, dass dann, wenn der Versicherte während der Dauer der Arbeitslosigkeit Leistungen der Gesetzlichen Unfallversicherung erhält, der Unfallversicherungsträger ein verstärktes Interesse an einer baldigen Vermittlung hat und schon deshalb die berufliche Wiedereingliederung forcieren wird.

\section{Reaktionen bei Veröffentlichung des Entwurfs}

Als der Arbeitsentwurf im Mai 2007 vom BMAS in die Öffentlichkeit gebracht wurde, hagelte es Proteste - und zwar aus unterschiedlichen Gründen aus allen Lagern. Die Bundesvereinigung der Deutschen Arbeitgeberverbände ist enttäuscht, hatte man sich doch von der Reform Kostensenkungen auch in Form des Wegfalls der Wegeunfälle versprochen. Tatsächlich gibt es seitens des BMAS offizielle Kostenschätzungen zu den voraussichtlichen Ausgaben, aus der Begründung sei zitiert:

„treten in den ersten 30 Jahren Mehrausgaben auf, danach langfristig Minderausgaben. Die Mehrausgaben steigen anfangs schnell auf fast 400 Mio. Euro/Jahr an, um dann langsam zurückzugehen. $\mathrm{Ab}$ etwa 2040 beginnt die Phase der Minderausgaben, die langsam anwachsen auf etwa 30.0 Mio €/Jahr“.

Die Gewerkschaften sehen ebenfalls Schlechterstellungen für ihre Mitglieder. Erhält z. B. nach altem Recht ein Versicherter infolge Lärmschwerhörigkeit ohne Gehaltseinbuße eine Rente in Höhe von $20 \%$, sind dies bei angenommenen 18.00o EUR Jahresarbeitsverdient (JAV) 200 EUR/Monat. Nach neuem Recht würde er lediglich einen Gesundheitsschadensausgleich von 50 EUR/Monat erhalten. Bei solch niedrigen Beträgen sehen die Gewerkschaften - wie ich meine 
nicht zu Unrecht - die finanziellen Anreize für Prävention geschmälert. Auch wehrt man sich dagegen, dass trotz Hinwendung zum konkreten Schadensausgleich generell Einkommensverluste bis $10 \%$ nicht berücksichtigt werden. So gesehen werden vermeintliche alte Ungerechtigkeiten durch neue ersetzt.

Auf Verwaltungsseite stößt der Gesetzentwurf ebenfalls auf wenig Gegenliebe. Konkrete Schadensberechnung ist mit individueller Verdienstermittlung und daher mit einem erheblichen Zusatzaufwand verbunden. Dabei geht es um die jährlich wiederkehrende Ermittlung des entgangenen Verdiensts und um die Feststellung, welche Tätigkeit zumutbar ist. Auch muss bei jeder Veränderung die Kausalität zwischen Unfall und Verdienstminderung geprüft werden - mit Sicherheit schwierig und streitbefangen. Als Verwaltung wehren wir uns auch gegen die Aussage, wir würden finanzielle Anreize benötigten, um eine wirksame berufliche Rehabilitation einschließlich Arbeitsvermittlung auf die Beine zu stellen. Die Arbeit unserer Berufshelfer wird von anderen Sozialversicherungszweigen und international anerkannt. Mit job.bg, einer Einrichtung zur Arbeitsvermittlung, sind die Berufsgenossenschaften seit jetzt 8 Jahren erfolgreich. Bereits jetzt haben die Berufsgenossenschaften einen Auftrag und den nehmen sie sehr ernst, sie benötigen dafür keine Anreize.

Der Deutsche Richterbund, der bekannt ist für seine seriösen Stellungnahmen, lehnt das vorgesehene neue Leistungsrecht ebenfalls ab. Er weist auf die hohe Akzeptanz des bisherigen Rentensystems bei den Versicherten hin und sieht diese im neuen Recht nicht mehr gewährleistet (beispielhafte Klagestatistik!). Es sei mit vielen Überprüfungs- und Änderungsbescheiden durch die Berufsgenossenschaften zu rechnen. Das neue Recht sei komplizierter, enthielte viele Unklarheiten und würde zu einer vermehrten Belastung der Gerichte führen.

\section{Resümee}

Angesichts dieser grundlegenden Kritik aus allen Bereichen ist das Schicksal des Arbeitsentwurfs zum Leistungsrecht äußerst fraglich. Offen wird von einer Ab-
Trauma Berufskrankh 2008 · 10[Suppl 2]:263-266 DOI 10.1007/s10039-007-1337-x

(c) Springer Medizin Verlag 2008

\section{H. Nolting \\ BG-Aktuell}

\section{Zusammenfassung}

Es sind grundlegende gesetzliche Neuerungen bezüglich der Organisation der gesetzlichen Unfallversicherung (GUV) und des Leistungsrechts geplant, Rahmenbedingungen dazu wurden 2006 in einem Eckpunktepapier festgelegt, jedoch von der Regierung bisher nicht umgesetzt. Die Berufsgenossenschaften jedoch haben bereits umfassende Veränderungen vorgenommen. So wurden Fusionen begonnen und vollzogen, Endziel sind 9 BG statt der im Eckpunktepapier geforderten 6. Die Politik hat dies akzeptiert und in den aktuellen Entwurf eines Unfallversicherungsreformgesetzes die Zahl 9 übernommen. Auch die politische Forderung nach Bildung eines gemeinsamen Dachverbands wurde von den Trägern der gesetzlichen Unfallversicherung kurzfristig umge-

setzt. Hier ist noch nicht klar, ob die Politik an ihrer Forderung der von den UV-Trägern nicht eingeplanten Verstaatlichung des Spitzenverbands festhält. Bezüglich der Finanzierung haben die Berufsgenossenschaften dem Gesetzgeber eine grundsätzliche und damit vom Eckpunktepapier abweichende Umgestaltung vorgeschlagen. Diese wurde aufgegriffen und ist Bestandteil des aktuellen Gesetzentwurfs. Das Leistungsrecht im sozialen Konsens zu reformieren, erscheint dagegen deutlich schwieriger, die bisherigen diesbezüglichen Planungen werden dargestellt.

\section{Schlüsselwörter}

Eckpunktepapier · BG-Fusion · Gemeinsamer Dachverband · Finanzierung · Leistungsrecht

\section{Update on employer's liability insurance associations}

\section{Abstract}

Plans have been made for basic legal innovations concerning the organization of statutory accident insurance and the right to social benefits. The general framework was established in the 2006 benchmark paper but it has not yet been implemented by the government. The employer's liability insurance associations have, however, already effected broad changes. Fusions were initiated and completed. The ultimate target is to form nine associations instead of the six prescribed in the benchmark paper. Policy-making authorities have agreed to this goal and incorporated the number nine into the current draft for the law reforming accident insurance. The political demand that a joint umbrella organization be formed was also quickly met by the accident insurance providers.
It is not yet clear whether policy-makers will adhere to the stipulation that the head organization be nationalized, which had not been planned by accident insurance providers. With respect to financing, the associations have suggested a fundamental reorganization, which diverges from the benchmark paper. This was accepted and is part of the current law draft. In contrast, the task of reforming the right to benefits and achieving social consensus appears to be considerably more difficult. Plans formulated up to now in this regard are presented.

\section{Keywords}

Benchmark paper - Fusion of employer's liability insurance associations . Joint umbrella organization · Financing $\cdot$ Right to benefits 
koppelung des Leistungsrechts vom Organisationsrecht bei der Reform gesprochen. Wenn aber das Leistungsrecht nicht mehr enthalten ist und man berücksichtigt, was die Berufsgenossenschaften bereits von sich aus umgesetzt haben und werden, bleibt von der so groß konzipierten Reform der Unfallversicherung wenig übrig.

\section{Neues aus der Rechtsprechung?}

\section{BG-Monopol}

Im Juli dieses Jahres hat das Landessozialgericht Sachsen durch Einzelrichterbeschluss ohne mündliche Verhandlung dem Europäischen Gerichtshof die Frage zur Entscheidung vorgelegt, ob die Pflichtmitgliedschaft des klagenden Unternehmens in einer BG gegen europäisches Recht verstößt. Hintergrund ist, dass der Bund der Steuerzahler und 3 Mittelstandsvereinigungen seit Mitte 2004 eine „Austrittskampagne“ gegen die Berufsgenossenschaften betreiben Sie haben diverse Unternehmer geworben, die ihren „Austritt“ aus der BG erklären und dann vor Gericht die vermeintliche Verfassungswidrigkeit des BG-Monopols festgestellt wissen wollen.

Rechtlich geht es um die Frage, ob das BG-Monopol gegen Wettbewerbsrecht und die Dienstleistungsfreiheit des EGVertrags verstößt. Hierzu ist zu bemerken, dass die italienische Unfallversicherung INAIL mit ihrer Pflichtversicherung ganz ähnlich geregelt ist wie die deutsche. Zur INAIL hat der Europäische Gerichtshof aber bereits vor einigen Jahren festgestellt, dass EU-Recht nicht verletzt ist. Bei fehlender Gewinnerzielungsabsicht, solidarischem Umlagesystem und staatlicher Aufsicht handelt es sich bei einem Unfallversicherungsträger um kein „Unternehmen “ im Sinne des EG-Vertrags. So sind auch bisher alle anderen Klagen gegen die Pflichtmitgliedschaft in einer BG abgewiesen worden. Erste Stimmen aus der Rechtswissenschaft gehen daher davon aus, dass die Berufsgenossenschaften „dem Verfahren sehr gelassen entgegensehen" können. Politisch gibt es zurzeit, wie bereits dargestellt, keine Mehrheit für Systemöffnungen oder Privatisierungen.

\section{Zuständigkeit der GUV}

Das Bundesverfassungsgericht hat eine für die Berufsgenossenschaften wichtige Aussage zu den Zuständigkeitsregelungen der gesetzlichen Unfallversicherung getätigt. Immer wieder wird den Berufsgenossenschaften vorgehalten, dass vergleichbare Unternehmen unterschiedlichen Berufsgenossenschaften mit unterschiedlichen Beiträgen zugeordnet werden würden.

Vor 4 Jahren hatte ein Leiharbeitsunternehmen gegen den aus seiner Sicht ungerecht festgesetzten Beitrag geklagt. Das Bundesverfassungsgericht aber nahm die Beschwerde erst gar nicht zur Entscheidung an. Die gesetzlichen Zuständigkeitsregelungen seien hinreichend bestimmt. Leiharbeitnehmer unterlägen aufgrund ihrer wechselnden Arbeitsstellen einem besonderen Risiko. Daher sei es auch nicht zu beanstanden, wenn direkt beim Entleiher angestellte Mitarbeiter bei einer anderen BG versichert seien und dort ein geringerer Beitrag für sie anfalle.

\section{Durchgangsärzte}

Stellt ein Durchgangsarzt im Zusammenhang mit einem Arbeitsunfall eine falsche Diagnose, kann dies einen Amtshaftungsanspruch des Versicherten gegen den Unfallversicherungsträger begründen. Denn der Arzt wird hier als Vertreter des Unfallversicherungsträgers tätig. Derzeit sind mehrere solcher Klageverfahren gegen Unfallversicherungsträger anhängig bzw. angedroht worden.

In einem Fall des Schleswig-Holsteinischen Oberlandesgerichts hatte ein Durchgangsarzt eine Schultereckgelenksprengung Tossy III als Prellung behandelt - mit irreparablen Folgen. Die Versicherte verklagte den D-Arzt auf Schadensersatz und Schmerzensgeld. Das Oberlandesgericht wies die Klage zurück mit der Begründung, der Durchgangsarzt habe in Ausübung eines öffentlichen Amts gehandelt. Schadensersatzansprüche wären damit allein gegen die Unfallversicherungsträger zu richten.

Es ist abzuwarten, ob sich diese Rechtsprechung durchsetzt. Die Unfallversicherungsträger würden in einem solchen Fall bei dem Durchgangsarzt Regress nehmen. Schadensersatzansprüche wegen reiner
Behandlungsfehler ohne primär falsche Diagnose bleiben zivilrechtlich geregelt und sind direkt im Verhältnis Patient-Arzt durchzusetzen.

\section{Auswirkungen der Reformen auf die Landesverbände}

Die DGUV nimmt sich aktuell dieser Frage an. Nach den vorliegenden Konzepten werden Strukturen gestrafft. Mitgliederversammlungen und Vorstände werden die Landesverbände voraussichtlich nicht mehr haben. Ab 2008 wären die einzelnen Landesverbände dann regionale Abteilungen der DGUV. Im Außenverhältnis, also auch den Ärzten gegenüber, soll es jedoch keine Abstriche bei der Dienstleistung geben.

\section{Korrespondenzadresse \\ H. Nolting \\ Landesverband Nordostdeutschland der gewerblichen Berufsgenossenschaften, Fregestraße 44, 12161 Berlin h.nolting@berlin.lvbg.de}

Interessenkonflikt. Der korrespondierende Autor gibt an, dass kein Interessenkonflikt besteht. 